\title{
The composition of liquid atmospheric pressure matrix-assisted laser desorption/ionization matrices and its effect on ionization in mass spectrometry
}

Article

Accepted Version

Creative Commons: Attribution-Noncommercial-No Derivative Works 4.0

Ryumin, P. and Cramer, R. (2018) The composition of liquid atmospheric pressure matrix-assisted laser

desorption/ionization matrices and its effect on ionization in mass spectrometry. Analytica Chimica Acta, 1013. pp. 43-53. ISSN 1873-4324 doi: https://doi.org/10.1016/j.aca.2018.01.070 Available at https://centaur.reading.ac.uk/75541/

It is advisable to refer to the publisher's version if you intend to cite from the work. See Guidance on citing.

To link to this article DOI: http://dx.doi.org/10.1016/j.aca.2018.01.070

Publisher: Elsevier

All outputs in CentAUR are protected by Intellectual Property Rights law, including copyright law. Copyright and IPR is retained by the creators or other copyright holders. Terms and conditions for use of this material are defined in the End User Agreement. 


\section{CentAUR}

Central Archive at the University of Reading

Reading's research outputs online 


\title{
The composition of liquid atmospheric pressure
}

\section{matrix-assisted laser desorption/ionization matrices}

\author{
and its effect on ionization in mass spectrometry
}

\author{
Pavel Ryumin and Rainer Cramer* \\ Department of Chemistry, University of Reading, Whiteknights, Reading RG6 6AD, United \\ Kingdom
}

Declarations of interest: None

\section{*Corresponding Author}

Address:

Department of Chemistry, University of Reading, Whiteknights, Reading RG6 6AD, United Kingdom. Fax:+44 118378 6331; Tel: +44 118378 4550; E-mail: r.k.cramer@reading.ac.uk 


\begin{abstract}
New liquid atmospheric pressure (AP) matrix-assisted laser desorption/ionization (MALDI) matrices that produce predominantly multiply charged ions have been developed and evaluated with respect to their performance for peptide and protein analysis by mass spectrometry (MS). Both the chromophore and the viscous support liquid in these matrices were optimized for highest MS signal intensity, $\mathrm{S} / \mathrm{N}$ values and maximum charge state. The best performance in both protein and peptide analysis was achieved employing light diols as matrix support liquids (e.g. ethylene glycol and propylene glycol). Investigating the influence of the chromophore, it was found that 2,5-dihydroxybenzoic acid resulted in a higher analyte ion signal intensity for the analysis of small peptides; however larger molecules $(>17 \mathrm{kDa})$ were undetectable. For larger molecules, a sample preparation based on $\alpha$-cyano-4-hydroxycinnammic acid as the chromophore was developed and multiply protonated analytes with charge states of more than 50 were detected. Thus, for the first time it was possible to detect with MALDI MS proteins as large as $\sim 80 \mathrm{kDa}$ with a high number of charge states, i.e. $\mathrm{m} / \mathrm{z}$ values below 2000. Systematic investigations of various matrix support liquids have revealed a linear dependency between laser threshold energy and surface tension of the liquid MALDI sample.
\end{abstract}

\title{
Keywords
}

atmospheric pressure (AP) matrix-assisted laser desorption/ionization (MALDI), ionization, liquid matrices, mass spectrometry, multiply charged proteins, surface tension 


\section{Introduction}

Matrix-assisted laser desorption/ionization (MALDI)[1, 2] is one of the two main ionization techniques in modern biological mass spectrometry (MS). Similar to the other main ionization technique, electrospray ionization (ESI)[3, 4], MALDI MS has been successfully and sometimes complementary applied to a vast array of analytes, from labile glyco-/phosphopeptides/-proteins[5, 6] and DNA/RNA[7-9] to metabolites[10-13] and synthetic (macro)molecules such as synthetic polymers $[14,15]$.

Traditionally, MALDI is directly coupled to time-of-flight (TOF) MS, which due to its pulsed nature and virtually unlimited mass range is ideal for the detection of ions with high $\mathrm{m} / \mathrm{z}$ values as it is the case for large biomolecular ions generated from conventional MALDI samples. Highvacuum requirements as well as overall analytical sensitivity in such setups have defined state-ofthe-art sample preparation favouring solid crystalline samples with low vapour pressure.

However, decoupling of ion generation from mass analysis and performing MALDI at elevated or atmospheric pressure (AP) offer significant advantages of improved mass accuracy[16-18] and generally softer ionization[16-20]. Another advantage is the ability to easily swap ion sources and utilize ESI mass spectrometers, providing a wider choice of fragmentation techniques than what is available with the commonly employed axial MALDI-TOF instruments. Although a few axial MALDI-TOF mass spectrometers also offer (high-energy) collision-induced dissociation (CID) MS/MS analysis[21], the vast majority of axial MALDI-TOF instruments rely on metastable postsource decay (PSD), which in such instruments typically leads to poorly resolved fragment ions[22, 23]. The introduction of AP-MALDI has also enabled its coupling to low-cost mass spectrometers[18]. 
In the last decade it was discovered that under certain conditions ESI-like multiply charged ions can be produced in AP-MALDI. Following earlier work where it was shown that IR-MALDI with its 'cooler' matrices can be advantageous in increasing the yield of multiply charged ions[24], Koenig et. al. reported the generation of predominantly multiply charged IR-MALDI ions from glycerol-based liquid samples for both peptides and proteins[25]. However, the MS sensitivity was in the high-picomole range with the typical drawbacks of IR-MALDI such as greater sample ablation and a more expensive and challenging optical setup compared to UV-MALDI. Later, Trimpin et. al. introduced laserspray ionization (LSI) for the generation of predominantly multiply charged ions from solid MALDI samples using an ultraviolet (UV) laser at high fluences[26]. Cramer et. al. introduced AP-UV-MALDI using liquid matrices[27], which also provide the advantage of low sample and analyte consumption at high ion yield and signal stability as previously reported for singly charged MALDI ions[23, 28-30]. These liquid matrices typically use solid MALDI matrices (chromophores) that absorb the laser energy and are dissolved in viscous support liquids with low volatility for extended analysis time. For both LSI and liquid APUV-MALDI the sensitivity was shown to be in the low-femtomole range[31, 32]. In the latter method this sensitivity was achieved with extremely low sample consumption allowing hours of signal acquisition and enabling novel strategies such as sample storage for prolonged periods of time and subsequent reanalysis[33].

It should be noted that at the same time ionic liquids were successfully introduced as MALDI matrices[34, 35]. However, none were reported to produce multiply charged ions and most of the reported ionic liquids applied to MALDI MS were not liquid under the given analytical conditions. Also, a new liquid (MA)LDI set-up was recently introduced that does not require the above mentioned matrix chromophores[36]. However, with this specific set-up the limit of detection 
(LOD) for the peptide melittin was reported to be $\sim 100 \mathrm{fmol}$, i.e. more than 2 orders of magnitude higher than the limits obtained in other studies with the addition of matrix chromophores[37]. The largest protein being detected with these liquid samples was myoglobin $(\sim 17 \mathrm{kDa})$ and it was noted that it was not possible to detect bovine serum album under the given conditions[36].

Here, new combinations of liquid UV-MALDI matrices are described that further advance the analytical capabilities of liquid UV-MALDI as introduced by Cramer et al.[27] and its ability to generate multiply charged peptide and protein ions. A typical liquid MALDI matrix satisfies four requirements: a chromophore to absorb the laser energy, an acid as a proton donor, a non-volatile (often highly viscous) support liquid to keep the sample liquid during analysis, and a volatile solvent to dissolve the chromophore/acid and facilitate the mixing of all components with the analyte. In previous AP-MALDI MS studies, employing glycerol-based MALDI sample preparations, and using UV or IR lasers, a limit of $17 \mathrm{kDa}$ was reported for the largest successfully analyzed biomolecule[25, 27, 36]. This limitation was linked to potential glycerol adduct formation[25]. Thus, particular attention was paid to finding new liquid matrix combinations that overcome these limitations. 


\section{Materials and Methods}

\subsection{Chemicals}

All chemicals were purchased from Sigma-Aldrich (Gillingham, UK) unless mentioned otherwise. Formic acid (FA) was obtained from Greyhound (Birkenhead, UK). HPLC-grade water $\left(\mathrm{H}_{2} \mathrm{O}\right)$ was purchased from Fisher Scientific (Loughborough, UK).

\subsection{MALDI sample preparation}

Various diols and triols were tested as the non-chromophore part (support liquid) of the liquid MALDI matrices for AP-MALDI MS analysis. Table 1 lists the 15 compounds that were investigated. Two liquid matrix solutions were prepared by dissolving $5 \mathrm{mg}$ of $\alpha$-cyano-4hydroxycinnamic acid (CHCA) in $1 \mathrm{~mL}$ of $50 \%$ acetonitrile (ACN) and $20 \mathrm{mg}$ of 2,5dihydroxybenzoic acid (DHB; 98\% purity) in $1 \mathrm{~mL}$ of $50 \% \mathrm{ACN}$, respectively, and subsequent sonication for $15 \mathrm{~min}$ at $30^{\circ} \mathrm{C}$. Aliquots of these solutions were then subsequently mixed with each of the support liquids listed in Table 1 in a ratio of 5:3 by volume to prepare 30 liquid MALDI matrices. In these preparations, any interfaces between liquid phases were broken by using a pipette tip and the solutions were thoroughly mixed. 
Table 1. Chemicals tested for their performance as viscous support liquids for AP-MALDI MS

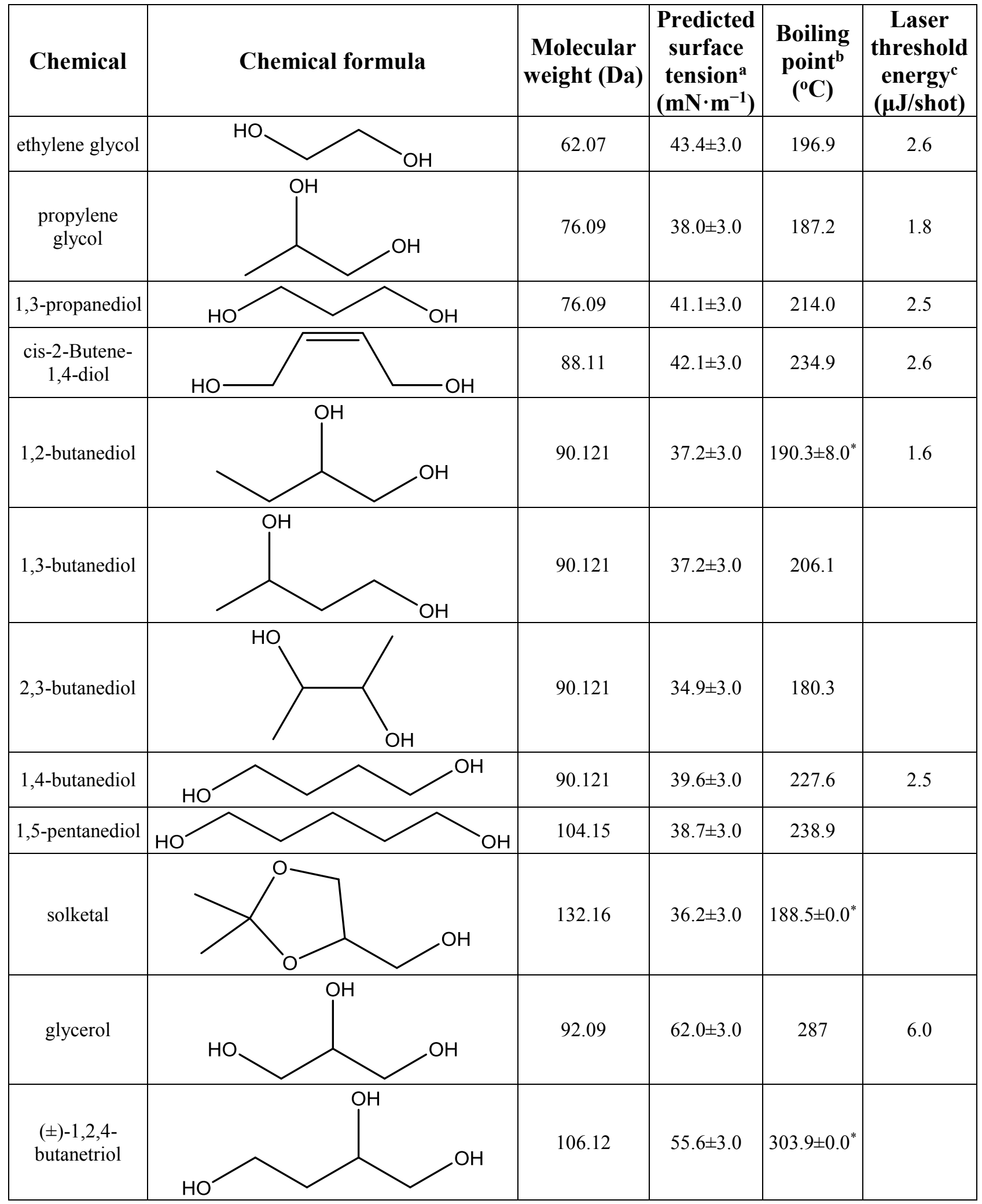




\begin{tabular}{|c|c|c|c|c|c|c|}
\hline $\begin{array}{c}\text { (S)-(-)-1,2,4- } \\
\text { butanetriol }\end{array}$ & \\
\hline hexylene glycol
\end{tabular}

${ }^{a}$ Surface tension at $20^{\circ} \mathrm{C}$. Values were obtained from ACD/Labs prediction on www.chemspider.com. ${ }^{\mathrm{b}}$ All values marked with ${ }^{*}$ were obtained from $\mathrm{ACD} /$ Labs prediction on www.chemspider.com. Other values are from the CRC Handbook of Chemistry and Physics by Haynes et al.[38]. ${ }^{\mathrm{c}}$ Laser threshold energy values were measured for MALDI samples containing the support liquid as described in 2.3 and 3.3.

2-Nitrophloroglucinol (NPG), CHCA, DHB, ferulic acid, sinapinic acid, and 2acetylphloroglucinol were used for investigating the chromophore part of the liquid MALDI matrices. A support liquid solution was prepared by adding $5 \mathrm{~mL}$ of $50 \%$ ACN to $3 \mathrm{~mL}$ of ethylene glycol. A total of 6 liquid MALDI matrices were prepared by dissolving $10 \mathrm{mg}$ of each chromophore in the support liquid solution.

All analytes were dissolved in $0.1 \%$ FA. An aliquot of $0.5 \mu \mathrm{L}$ of the liquid MALDI matrix was mixed with $0.5 \mu \mathrm{L}$ of the analyte solution on the target plate and left drying at room temperature for $30 \mathrm{~min}$.

\subsection{Sample preparation for surface tension and MALDI laser energy threshold studies}

A volume of $30 \mathrm{~mL}$ of each DHB-glycerol and DHB-ethylene glycol solution was prepared by individually mixing DHB with each support liquid in a ratio of $10 \mathrm{mg}$ to $1 \mathrm{~mL}$ and heating the mixture to $60^{\circ} \mathrm{C}$ in a temperature-controlled water bath. After this, the two liquid matrix solutions were vortexed until the DHB was completely dissolved, and a volume of $400 \mu \mathrm{L}$ of a $0.1 \%$ FA solution of $500 \mathrm{ng} / \mu \mathrm{L}$ LeuEnk was mixed with each of the two liquid matrix solutions. These two 
liquid matrix/analyte solutions were then used to prepare a range of liquid MALDI samples with different surface tensions. For this, the ethylene glycol-based solution was mixed with the glycerolbased solution in ratios of 5:0, 4:1, 3:2, 2:3, 1:4, 0:5. Aliquots of $50 \mu \mathrm{L}$ of each mixture were taken for the laser energy threshold measurements with $1 \mu \mathrm{L}$ being spotted on the MALDI target plate. The remainders of the mixtures were used for the surface tension measurements as described below.

\subsection{MALDI MS instrumentation}

A Synapt G2-Si mass spectrometer (Waters Corporation, Wilmslow, UK) was modified with an in-house developed AP-MALDI ion source, described in detail elsewhere[32], and used to acquire all MS data. The ion source is equipped with an extended heated ion transfer tube, which has been designed to provide an additional control over the MALDI plume desolvation conditions. In this setup, a counter nitrogen gas flow can be introduced at the interface of the heated ion transfer tube, where it joins the MS inlet, allowing an adjustment of the infused gas flow velocity. A heating power of $25 \mathrm{~W}$ was applied to a resistance wire, heating the ion transfer tube. To control the capillary temperature and MALDI plume residence time the counter gas flow was adjusted in the range of $0-180 \mathrm{~L} / \mathrm{h}$, providing capillary temperatures of $250-350^{\circ} \mathrm{C}[32]$. The instrument's ion block temperature was set to $80^{\circ} \mathrm{C}$, and the cone voltage was set to $40 \mathrm{~V}$. A nitrogen laser $(\lambda=337 \mathrm{~nm}$; MNL 103 LD; LTB Lasertechnik GmbH, Berlin, Germany) was used with a neutral density filter driven by a stepper motor, which was installed on the beam path to attenuate the laser energy. The laser pulse energy was varied in the range of $1-30 \mu \mathrm{J}$ per shot, and the laser beam was focused to a diameter of approximately $100-150 \mu \mathrm{m}$. The laser pulse repetition rate was $30 \mathrm{~Hz}$. The distance between the ion transfer tube and MALDI target plate was $3 \mathrm{~mm}$ and the target plate potential was set to $4 \mathrm{kV}$. Spectra were acquired with the drift time recording in the ion mobility separation (IMS) 
cell enabled and the IMS Wave Velocity was set to $650 \mathrm{~m} / \mathrm{s}$ while the IMS Wave Height was set to $40 \mathrm{~V}$.

\subsection{Data processing}

The acquired MS data was converted to the mzML format using the ProteoWizard software package (version 3.0.10730)[39]. In-house developed software based on the pyteomics software library (version 3.4.1)[40, 41] was used to extract and integrate signal intensities of analyte ion peaks. For plotting spectra, a matplotlib library (version 1.5)[42] was used and the acquired signal at each $\mathrm{m} / \mathrm{z}$ value was combined from multiple scans by binning the whole $\mathrm{m} / \mathrm{z}$ range and summing the ion signal corresponding to these bins. The bin size was set to $1 \mathrm{Da}$.

\subsection{Laser energy threshold measurements}

To control the laser energy, a driver for the stepper motor of the laser energy attenuator was developed and bridged with the MS acquisition software using a top-level layer in the Waters Research Enabled Software (WREnS; Waters Corporation). The WREnS script written for adjusting the attenuator's position allowed for the automated adjustment of the laser energy attenuation during MS acquisition in small steps with a specified acquisition time during which the laser energy is set to a certain value and a time delay between the acquisitions, allowing the system to change the laser energy value and avoid any carry-overs. As a result, this setup provided fully automated recording of an ion chromatogram, correlating the laser pulse energy values (for all time windows) to the MS ion signal intensities obtained. MS data acquisitions with durations of $5 \mathrm{~s}$ and $10 \mathrm{~s}$ for each attenuation step were used with a delay of $3 \mathrm{~s}$ between them. Each attenuation step (100 stepper motor steps) corresponded to a laser pulse energy step of $\sim 1.0-1.5 \mu \mathrm{J}$. The exact laser energy per shot was measured by an energy meter (Molectron J9LP; Coherent Inc., Santa 
Clara, USA) after each acquisition. A total of 5 data sets were acquired for each sample and the integrated ion intensity was calculated for each laser energy step. These values were used to determine the laser energy threshold for analyte ion production using the criterion that all data points acquired with a laser energy above or equal to the threshold value have an integrated ion signal intensity of more than 500 arbitrary units, which correspond to $\sim 15$ ion counts per second. This absolute ion count threshold level was applied since the noise level was always between 0 100 arbitrary units ( $\leq 3$ ion counts per second) in the immediate analyte $\mathrm{m} / \mathrm{z}$ range, thus guaranteeing a minimum signal-to-noise ratio of 5 as well as avoiding any strong influence of a changing noise level due to changes of the atmosphere in the ion source.

\subsection{Surface tension measurement}

The surface tension values of the samples were measured by a tensiometer (K100; Krüss, Hamburg, Germany) at $20^{\circ} \mathrm{C}$ using the Wilhelmy plate method. The plate was initially submerged in the sample and 10 consecutive measurements were then acquired over 2.5 minutes. 


\section{Results and discussion}

\subsection{Effect of the matrix composition and instrument parameters on protein analysis}

Some of the challenges with the analysis of large macromolecules are unwanted fragmentation during the ionization process and adduct formation. Both processes can lead to a depletion of the parent ion signal, invariably decreasing the signal-to-noise $(\mathrm{S} / \mathrm{N})$ level, and also resulting in unresolved spectra.

Figure 1a shows a liquid AP-UV-MALDI mass spectrum of BSA, illustrating the ion signal quality for larger biomolecules achievable with previously devised liquid AP-UV-MALDI MS sample preparation methods. In the presented study, one of the objectives was to find a liquid MALDI sample preparation suitable for the analysis of large macromolecules. A particular focus was on the optimization of the viscous support liquid and the chromophore, as these agents unlike the volatile solvents are present in the sample during MS analysis.

Here, it should be noted that a recent study by Koch et al. has shown that a matrix chromophore is not needed for detecting multiply charged peptide and protein ions, although arguably at the expense of sensitivity[36]. In the present study, initial tests revealed that a lower LOD and sample consumption were obtainable with the addition of matrix chromophores, which is also in agreement with the results presented later with regard to changing the added matrix chromophore. For sample droplets without matrix chromophores, it was found that substantial peptide ion signals were only obtainable at the edge of the sample droplet and with much greater sample consumption, which is also in agreement with other studies on lipids[43]. Unfortunately, Koch et al. did not provide any information on the sample consumption. However, their highly unusual ion source geometry with the sample being presented at an equally unusual angle to the MS analyzer ion beam 
trajectory and the relatively large focal spot size, which was 10-100 times larger than in this study, make a comparison difficult.
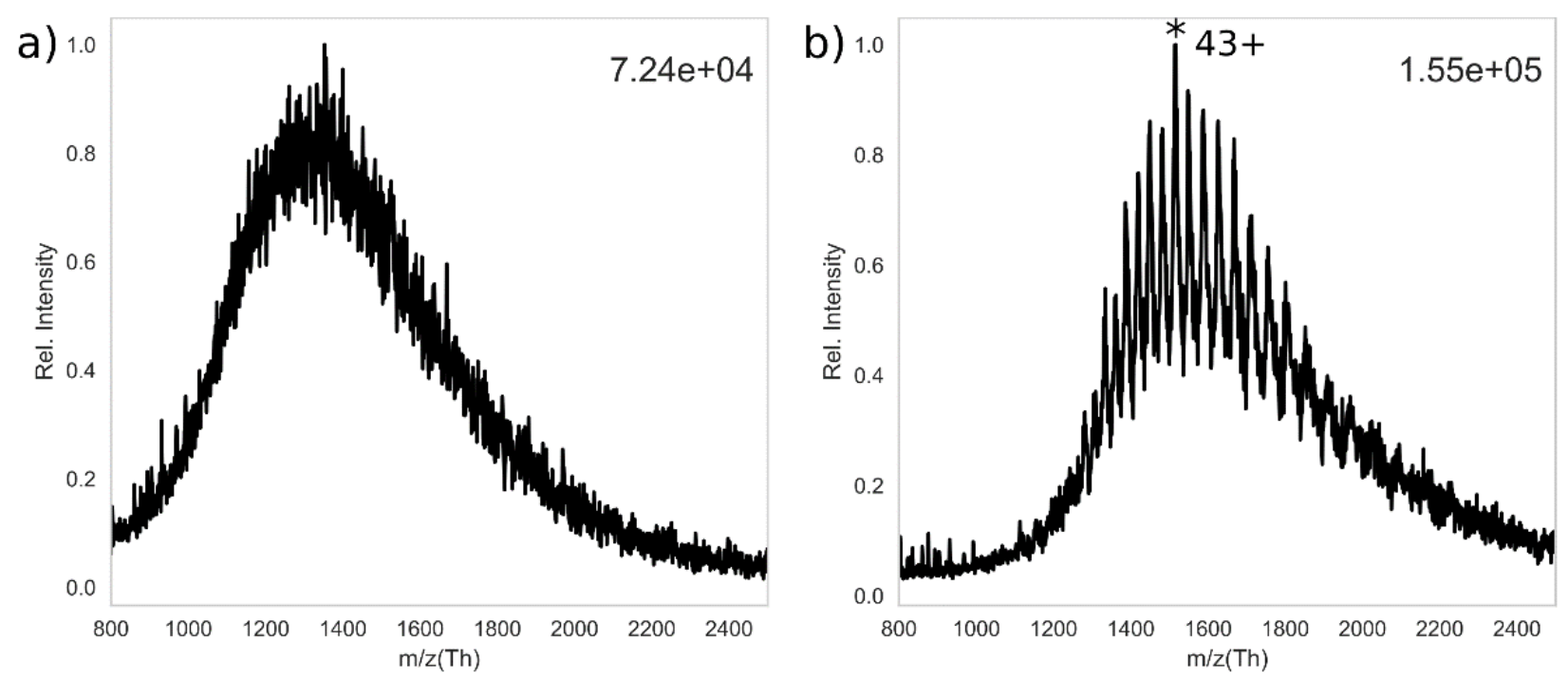

Figure 1. a) Liquid AP-UV-MALDI MS spectrum of BSA acquired using a liquid matrix with DHB as the chromophore and glycerol as the viscous support liquid. b) Liquid AP-UV-MALDI MS spectrum of BSA acquired using a liquid matrix based on CHCA as the chromophore and ethylene glycol as the viscous support liquid. In each case a total of $12.5 \mathrm{pmol}$ of protein was spotted using a $1-\mu \mathrm{L}$ MALDI sample droplet. The most intense analyte ion peak is marked with an asterisk and labeled with its charge state.

In our initial experiments two chemicals were used as the laser energy-absorbing UV chromophores: DHB and CHCA. The results obtained with CHCA were more reproducible, showing well-resolved MS spectra of small proteins even under conditions that were not fully optimized. Another important tuning parameter was the counter gas flow in the heated ion transfer tube. In our setup, the counter gas flow when optimized for peptide analysis increases the 
temperature as well as the MALDI plume residence time in the heated ion transfer tube, hence promoting more efficient plume desolvation[32]. In contrast to peptide analysis any counter gas flow was found to be detrimental for the analysis of larger molecules. Finally, in the attempt to improve protein analysis performance, glycerol as the liquid support in previously reported sample preparations was replaced by ethylene glycol, resulting in well-resolved BSA mass spectra as demonstrated in Figure 1b.

These findings led to the investigation of 14 other chemicals listed in Table 1 as (viscous) support liquids for liquid AP-UV-MALDI sample preparations, as well as an investigation of other known MALDI matrix chromophores and a search for optimum desolvation conditions.

In a first experiment, a set of matrices using different viscous support liquids from Table 1 was prepared as described in 2.2 with CHCA used as the chromophore. The heating power applied to the ion transfer tube was $25 \mathrm{~W}$, and no counter gas flow (ion transfer tube temperature of $250^{\circ} \mathrm{C}$ ) was used for these measurements. The laser pulse energy was set to $30 \mu \mathrm{J}$ per shot. Three MALDI samples were spotted for each tested support liquid. Each spotted sample was irradiated for $15 \mathrm{~s}$ and the data from the three samples were combined into one mass spectrum. Figure S1 (Supplementary Material) shows the results of this experiment for human carbonic anhydrase I prepared at a concentration of $10 \mathrm{mM}$ for the spotted MALDI sample droplets of $1 \mu \mathrm{L}$. Likewise, Figure S2 (Supplementary Material) shows the results of a similar experiment for a BSAcontaining sample droplet prepared at a concentration of $12.5 \mathrm{mM}$. These results show that for a smaller protein, such as carbonic anhydrase I, it is possible to acquire a charge state-resolved protein mass spectrum with all tested diols. However, the use of smaller molecules such as ethylene glycol and propylene glycol for the viscous support liquid generally results in greater analyte ion signal intensity and/or S/N. For BSA analysis, only the sample preparation based on diols resulted 
in a well-resolved spectrum, and larger chemicals, e.g. triols, were found unsuitable as liquid support for such analysis.

A similar study as described above was also carried for chromophores. Six chromophores (NPG, CHCA, DHB, 2-acetophloroglucinol, ferulic acid, and sinapinic acid) were investigated for the analysis of human carbonic anhydrase I, with all acquisition parameters set the same as for the experiment with the viscous support liquids apart from the sample preparation. For the sample preparation, the second protocol described in 2.2 was used. The results of this experiment are shown in Figure 2. This set of measurements shows that both chromophores CHCA and NPG give comparable performance for the analysis of large molecules. The spectra acquired with the matrices using these chromophores display substantially higher $\mathrm{S} / \mathrm{N}$ and higher charge state ions compared to the spectra acquired with the matrices based on other chromophores. As the CHCA sample preparation provides double the analyte ion signal intensity than NPG, it became the sample preparation of choice of all tested preparations for the analysis of large molecules. The chromophores 2-acetylphlouroglucinol, ferulic acid and sinapinic acid also allowed analyte detection, albeit with a considerably lower charge state and signal intensity. The use of DHB as a chromophore, however, led to a completely unresolved charge state distribution (see Figure 2). 

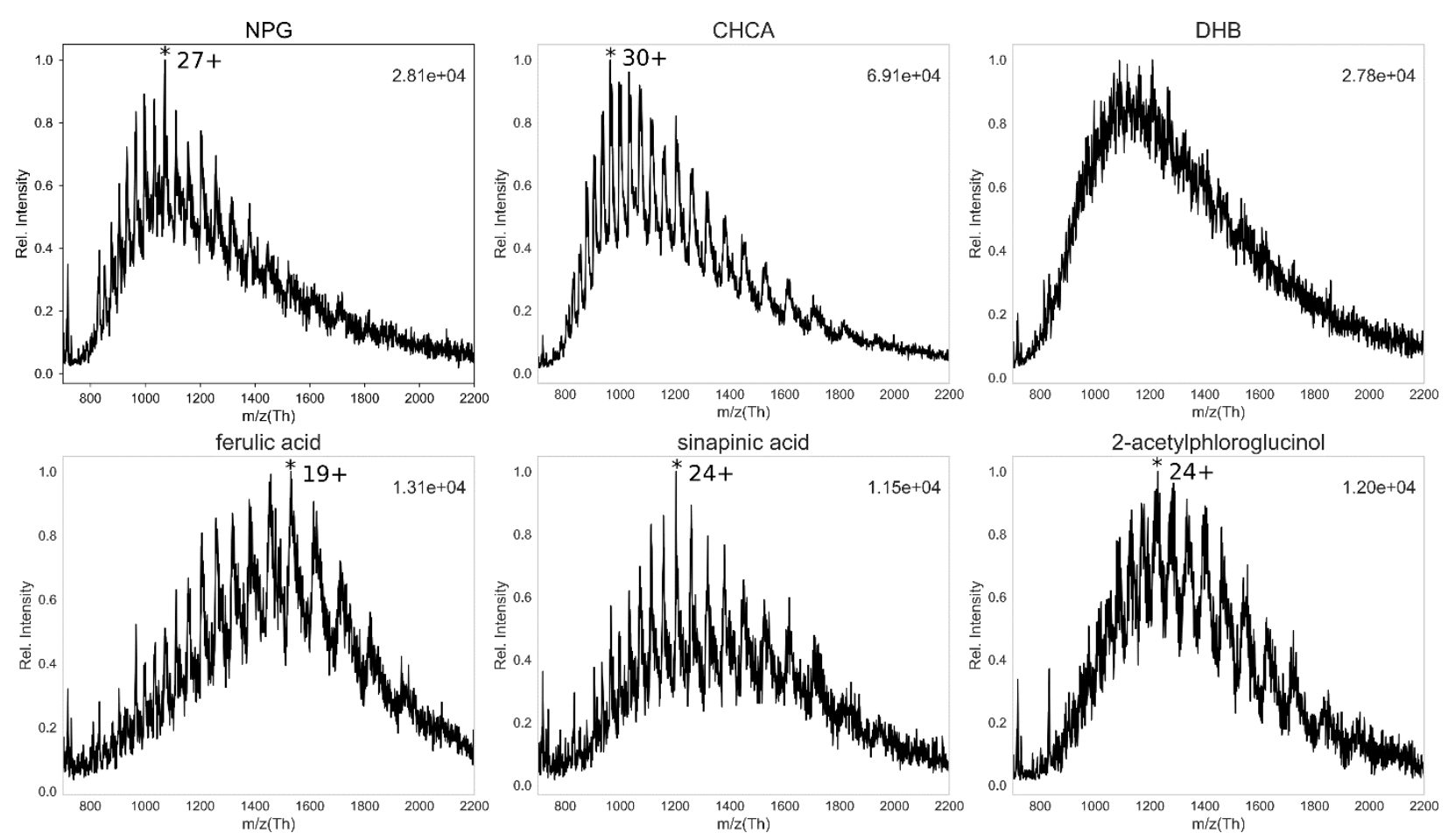

Figure 2. Liquid AP-MALDI mass spectra of human carbonic anhydrase I using different chromophores. In each case a total of $10 \mathrm{pmol}$ of protein was spotted in a $1-\mu \mathrm{L}$ MALDI sample droplet, the counter flow was set to $0 \mathrm{~L} / \mathrm{h}$ and ethylene glycol was used as the viscous support liquid. The most intense analyte ion peak is marked with an asterisk and labeled with its charge state.

In a third experiment, mass spectra of human carbonic anhydrase I were acquired at different counter gas flow settings. For this experiment the sample preparation was the same as for the investigation of viscous support liquids with ethylene glycol as the viscous support liquid. The counter gas flow was adjusted in $30 \mathrm{~L} / \mathrm{h}$ steps in the range of $0-180 \mathrm{~L} / \mathrm{h}$ while all other instrument parameters were kept the same as before. The results can be found in Figure 3, showing that with the increase of the counter gas flow the spectral quality monotonically degrades, i.e. S/N, absolute signal intensity, and charge states gradually decrease. 

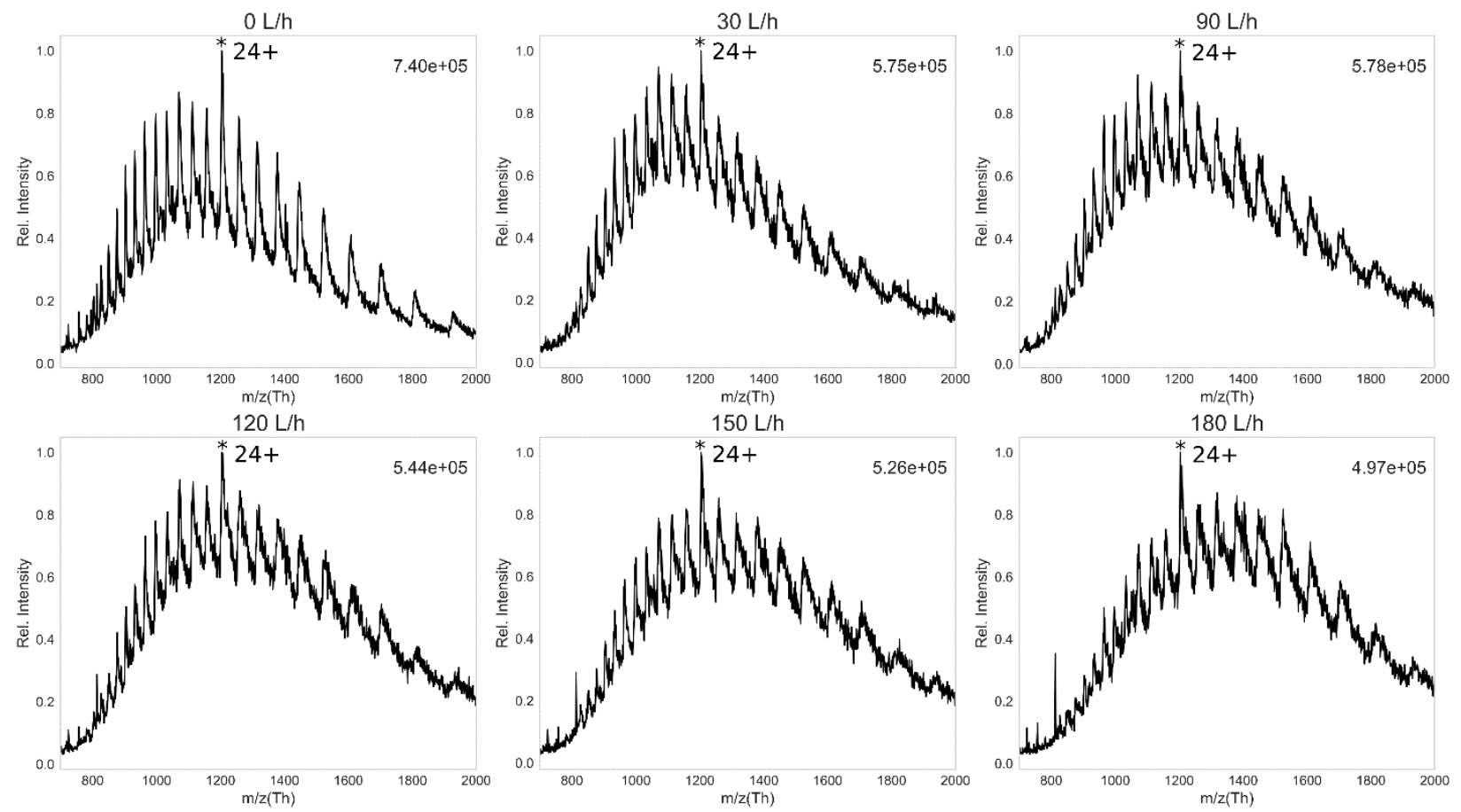

Figure 3. Liquid AP-MALDI MS ion signals of human carbonic anhydrase $\mathrm{I}$ in response to different counter gas flow values. For each spectrum, a total of $10 \mathrm{pmol}$ of protein was spotted in a $1-\mu \mathrm{L}$ MALDI sample droplet. For these experiments 5mg CHCA was dissolved in 50\% ACN and mixed with ethylene glycol in a 5:3 (v:v) ratio. The most intense analyte ion peak is marked with an asterisk and labeled with its charge state.

\subsection{Analysis of human apo-transferrin}

All of the described enhancements were then applied to the measurement of human transferrin ( $\sim 80 \mathrm{kDa})$. The liquid AP-MALDI MS results for $50 \mathrm{pmol}$ of human transferrin spotted on the MALDI target are shown in Figure 4. For this acquisition, the liquid matrix was prepared with $5 \mathrm{mg} / \mathrm{mL}$ of CHCA and the addition of $60 \%$ ethylene glycol (by volume; see first paragraph of section 2.2). Three samples were spotted, each sample was irradiated for $\sim 4 \mathrm{mins}$ and the collected 
data was combined. To the authors' knowledge, this is the first published MS data of a predominantly ESI-like multiply charged protein over $75 \mathrm{kDa}$ obtained by a laser-based desorption technique. It should also be noted that in contrast to common guidelines for solid MALDI MS, CHCA-based liquid MALDI samples were superior for the analysis of larger proteins compared to DHB-based liquid MALDI samples.

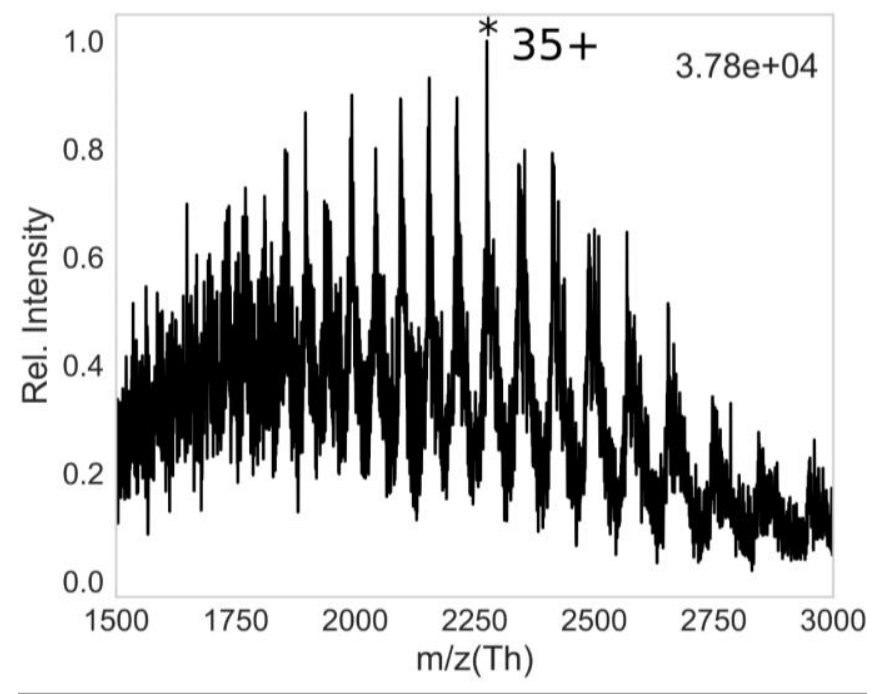

Figure 4. Liquid AP-MALDI mass spectrum of human apo-transferrin. The spectrum was acquired using CHCA and ethylene glycol for the liquid matrix and a counter gas flow value set to $0 \mathrm{~L} / \mathrm{h}$. A total of 50pmol of protein was spotted using a 1- $\mu$ L MALDI sample droplet. The most intense analyte ion peak is marked with an asterisk and labeled with its charge state.

Overall, these data suggest that the analysis of large molecules $(>50 \mathrm{kDa})$ is possible in AP-MALDI MS using liquid matrices, but the performance greatly depends on both the MALDI sample composition and MS acquisition parameters. In this investigation, the hypothesis that the viscous support liquid easily forms adducts with large analytes could not be confirmed. In fact, with the optimization of other parameters, it was possible to extend the mass range of analysis to the mass 
of human carbonic anhydrase I without the need to change the composition of the liquid MALDI sample, i.e. still allowing the use of glycerol. Importantly, in the experiments with smaller proteins, where the adduct peaks were well resolved, only salt cation adducts, and no support liquid compound adducts were detected (data not shown). For larger proteins with increased probability of multiple salt cation adduct formation, this can potentially lead to strong analyte ion signal suppression and wide peak tailing, which was observed in this study, and can effectively limit the detection of larger proteins. The MALDI sample composition, including the type of chromophore and viscous support liquid and their concentrations, can arguably have a substantial effect on the extent of adduct peak formation through their influence on the absorption of the laser energy and the ablated plume composition. As a consequence, the ablated/desorbed droplet sizes in the plume and their composition during the initial stages of the MALDI process can vary substantially. Similar to the ESI process[44], it can be argued that the extent of cation adduct formation is dependent on the initial size of the droplet and its morphology. In the present study it was also observed that excessive heating in the ion transfer tube can lead to extensive salt cation adduct formation for peptides, which can be explained by thermal desorption of salt cations from the walls of the ion transfer tube and their gas phase reactions with the analyte ions. Thus, both optimum desolvation conditions and optimum composition and size of the generated droplets appear to be needed for obtaining quality spectra with low adduct formation. However, further experiments are needed to test this hypothesis.

\subsection{Effect of the matrix composition and instrument parameters on peptide analysis}

To test the performance of the newly developed matrices for peptide analysis, experiments were also carried out using angiotensin 1 and bradykinin. An extensive investigation of the optimal desolvation conditions for the liquid AP-MALDI MS analysis of these two peptides was previously 
undertaken[32], where it was found that for the given experimental setup a counter gas flow in the range of $100-210 \mathrm{~L} / \mathrm{h}$ is beneficial for the detection of multiply charged ions, providing improved detection limits, signal intensities and $\mathrm{S} / \mathrm{N}$ values. Similar experiments were also performed with the newly developed matrices employing ethylene glycol and propylene glycol as viscous support liquids. The results of these experiments (data not shown) agreed with the previous data, and therefore no additional investigation on the optimization of the desolvation conditions was performed. Thus, a counter flow value of $180 \mathrm{~L} / \mathrm{h}$ was used for all reported experiments with the other instrument parameters kept the same as in 3.1 .

First, liquid matrices employing the viscous support liquids of Table 1 were tested. The matrices were prepared using DHB as chromophore according to the protocol described in 2.2. All tested matrices were found suitable for the detection of multiply charged angiotensin 1 and bradykinin ions. Figure 5 shows the total ion intensities and average charge states of the angiotensin I ion signals obtained in this experiment. The acquired data clearly show that both ethylene glycol and propylene glycol offer superior performance to all other tested support liquids yielding more than 3 times the signal intensity of using the next best support liquid and more than 4 times that of using glycerol. The average charge state was also found to be slightly higher for these support liquids. In general, it was found that the MALDI matrices employing smaller diols as viscous support liquids provide higher signal intensity than the reference matrix employing glycerol. 

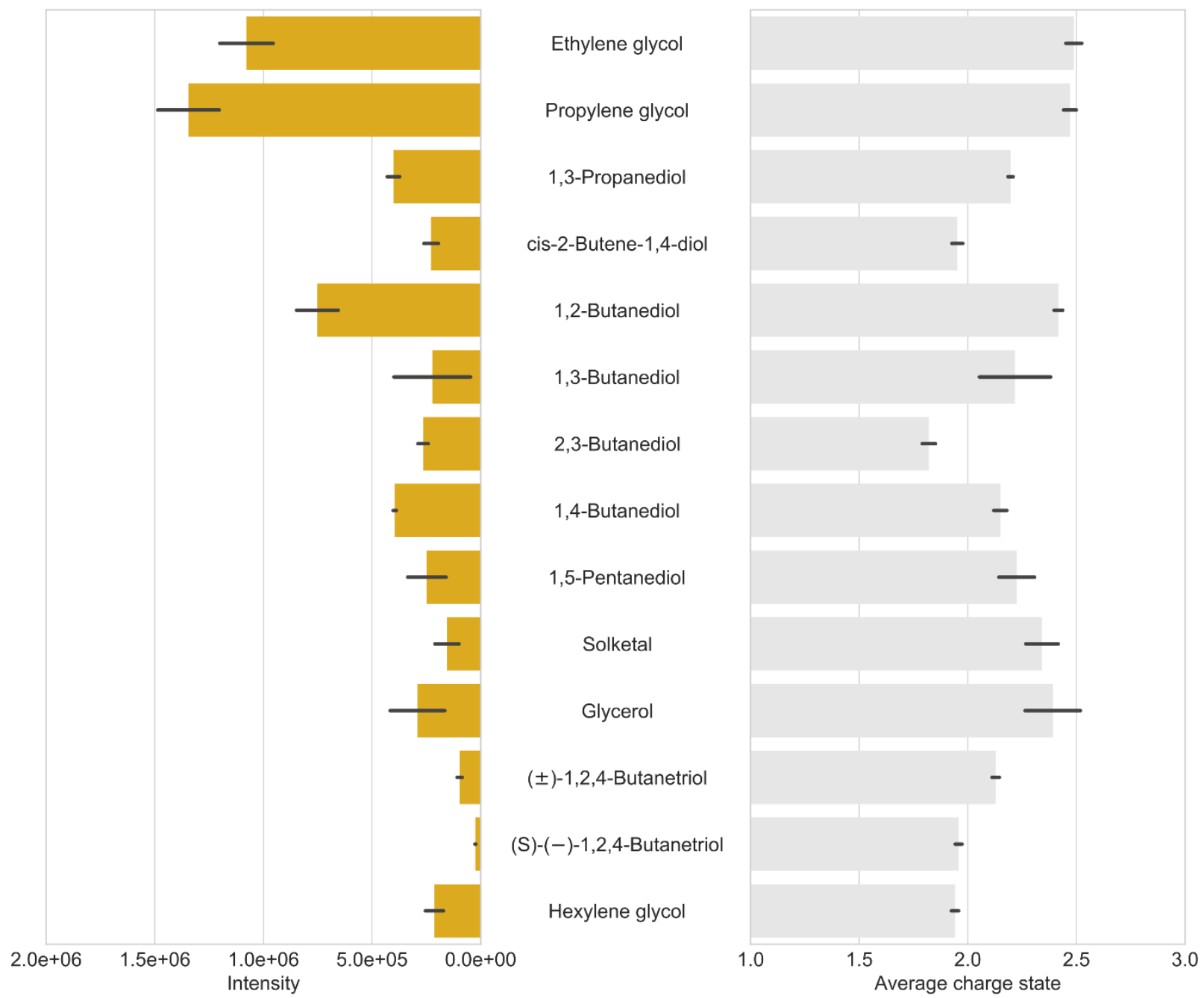

Figure 5. Angiotensin I ion signal intensities (left panel) and average charge states (right panel) in response to different support liquids. An equimolar two-peptide mixture of angiotensin I and bradykinin (10 pmol total peptide amount loaded) was analyzed by liquid AP-MALDI MS. Error bars represent the standard deviation $(n=3)$.

With respect to the LOD, ethylene glycol and propylene glycol performed similarly well as glycerol. One femtomole or less of peptide spotted in a 1- $\mu$ L MALDI sample droplet using DHB as the chromophore was sufficient to be detected. 
In another experiment the effect of the chromophore compound in MALDI sample preparation was studied for peptide analysis. The same chromophores as in 3.1 were also tested in this experiment. The sample preparation was identical to the protein analysis experiment with ethylene glycol as the viscous support liquid. The acquisition conditions were kept the same as in the experiment described above in this section. Figure 6 shows the results of this experiment, where the intensities of the triply (grey), doubly (dark yellow) and singly (brown) charged angiotensin I ions are displayed for all tested matrices. These data show that the widely used MALDI chromophores DHB and CHCA offer superior performance compared to all other tested chromophores. The matrices employing DHB also led to a notably higher doubly charged ion signal in our experiments, but the triply charged ion signal was the same compared to the one achieved with CHCA. The other tested chromophores provided detectable ion signal, but signal intensities were considerably smaller compared to DHB and CHCA. In the measurements with 2acetylphloroglucinol no singly charged ion signal was detected. 


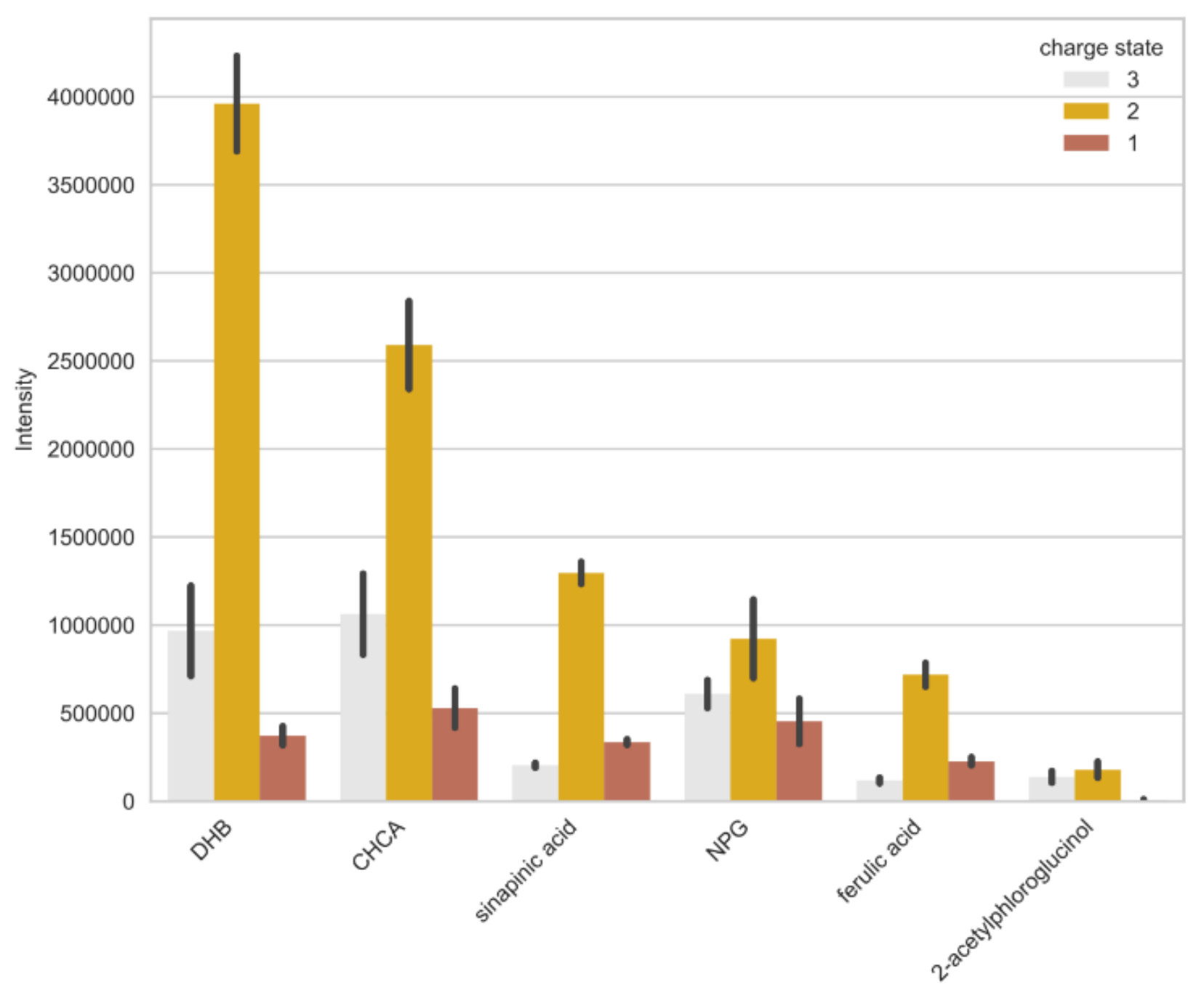

Figure 6. MS signal intensities of triply (grey), doubly (dark yellow), and singly (brown) charged angiotensin I ions in response to the use of different chromophores. An equimolar two-peptide mixture of angiotensin I and bradykinin (10 pmol total peptide amount loaded) was analyzed by liquid AP-MALDI MS. Error bars represent the standard deviation $(n=3)$.

\subsection{MALDI sample surface tension dependency}

During the study of different viscous support liquids, it was discovered that the MALDI samples prepared with support liquids of high surface tension required higher threshold laser energies for 
generating analyte ions than MALDI samples prepared with support liquids of low surface tension. To further investigate this behaviour, the laser energies at the ion detection threshold, i.e. the laser energies at which a chosen reporter ion signal disappeared based on a signal-to-noise ratio of below 5 , were recorded for each sample. These measurements were undertaken by desorbing from an area around the center of the sample droplet using the same geometrical alignment of the incoming laser beam on the liquid MALDI sample droplet in order to avoid differences due to the irradiated droplet surface area. The recorded laser energies were found to be in good direct correlation with the viscous support liquid's surface tension values taken from the literature (see Table 1). To further verify the hypothesis that the threshold laser energy for ion detection is dependent on the MALDI sample surface tension, the surface tension values of liquid MALDI samples prepared with the same chromophore (DHB) and analyte (bradykinin) but various viscous support liquids were directly measured. The results of these measurements are shown in Figure $7 \mathrm{a}$ and show a clear linear correlation between the threshold laser energy and the measured MALDI sample surface tension.

The assignment of the threshold laser energies in these experiments can be prone to potential inaccuracies due to the simultaneous recording of laser energies and analyte signals by the operator at a level of low ion signal/statistics. Also, a potential carry-over from a previous measurement may be registered as part of the ion signal, if the measurements are taken too fast during a downward (or upward) laser energy scan, thus distorting the recording of accurate values. To alleviate these shortcomings, a setup for the automated acquisition of each threshold laser energy measurement with a sufficient time interval between measurements was developed in addition to the above-mentioned criterion for the threshold determination. 
Another potential error source in investigating the dependency of the threshold laser energy on the surface tension is the MALDI sample drying on the target plate, which is difficult to control as the MALDI samples described in this study contain $>80 \%$ volatile solvents. Depending on the pressure and the viscous support liquids and volatile solvents used, a generally unknown fraction of each of the volatiles is still retained in the droplet at the time of the measurement. To some extent these differences between the viscous support liquids also explain the different deviations of the measured surface tension values for a liquid MALDI sample preparation in Figure 7a from the predicted surface tension values of the pure viscous support liquid in Table 1. While a relatively short drying time of a few minutes is usually sufficient for reproducible conventional liquid MALDI MS analysis, substantial amounts of volatile solvents can still be present and slowly evaporate over time. This situation is obviously not ideal for longer measurements in which the threshold laser energy of several (replicate) samples has to be determined.. As the composition of the droplet significantly affects its physical properties, measures need to be taken to ensure the same composition during both the surface tension and the MS threshold measurements. One way of achieving this is to minimize the amount of volatile solvents in the initial sample composition, e.g. through the use of the viscous support liquid as the main solvent for the UV chromophore and analyte. Such approach was employed with glycerol and ethylene glycol as both solvent and support liquid, and two DHB-based liquid matrices as well as four binary mixtures in different ratios were prepared as described in 2.3. In all these sample preparations, the volatile solvent content can be calculated to be $\leq 1 \%$ of the total MALDI sample volume. The results of these measurements are shown in Figure 7b, displaying a linear dependency of the threshold laser energy on the sample surface tension. 

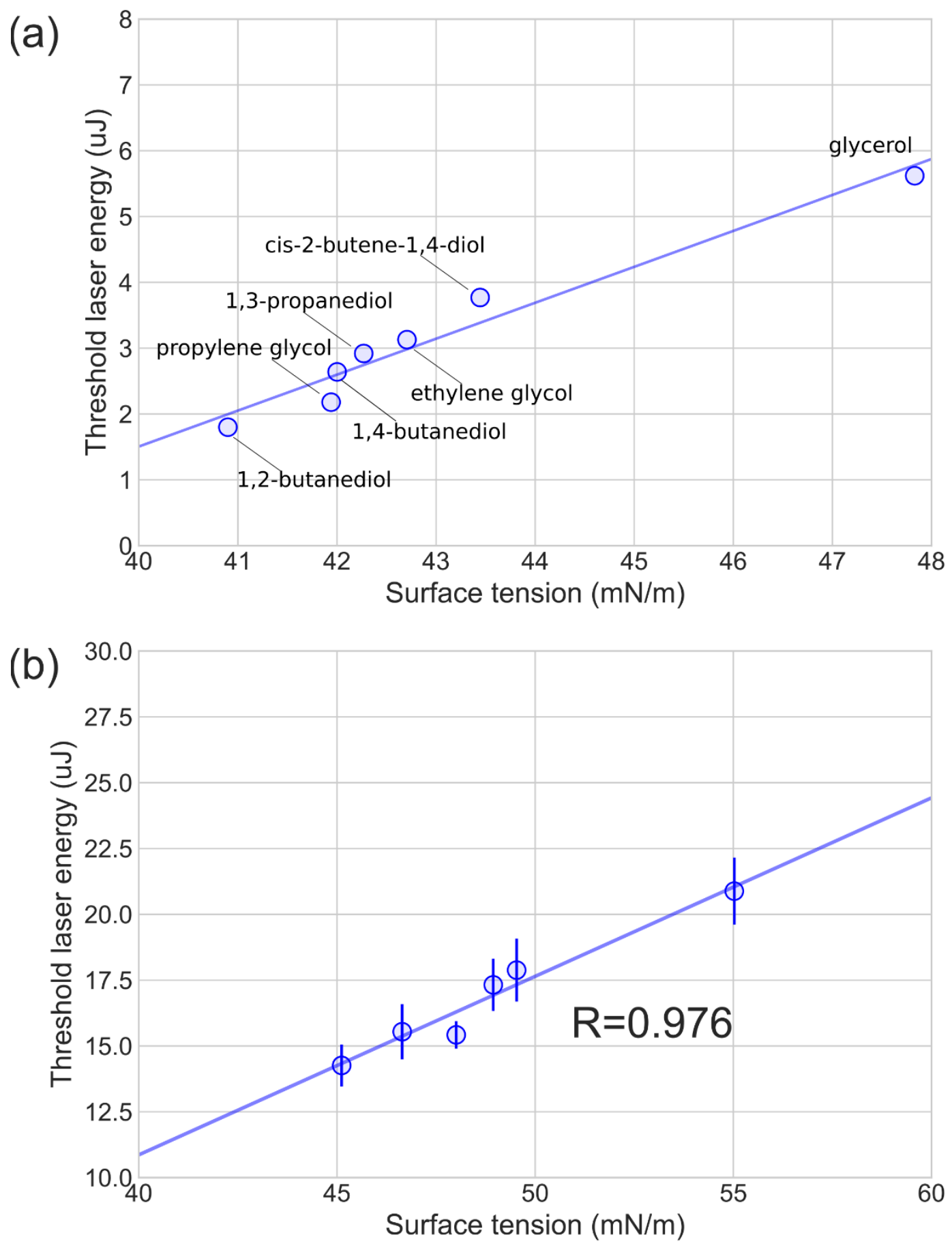
Figure 7. Threshold laser energy for MALDI MS ion detection as a function of sample surface tension using a) a 1:1 mixture of an analyte solution and a liquid MALDI matrix mixed on a target plate (labels correspond to the viscous support liquid used in the matrix preparation as described in 2.2) and b) a sample consisting of a binary mixture of ethylene glycol and glycerol doped with an analyte and DHB as UV chromophore. Error bars represent the standard deviations ( $\mathrm{n}=5$ for threshold laser energy measurements, and $\mathrm{n}=10$ for surface tension measurements).

Data supporting the results reported in this paper are openly available from the University of Reading Research Data Archive at http://dx.doi.org/10.17864/1947.102.

\section{Conclusions}

This study highlights the importance of sample preparation in liquid AP-MALDI MS. Newly developed liquid matrices capable of generating multiply charged ions were significantly enhanced by employing alternative viscous support liquids, such as ethylene glycol and propylene glycol, and chromophores tailored to the needs of the analyte, e.g. CHCA-based matrices for the analysis of large macromolecules. Together with optimized instrumental parameters this allowed the successful detection of predominantly multiply charged human apo-transferrin ions, the largest predominantly multiply charged protein so far reported to be analyzed by a laser-based desorption technique.

The newly discovered linear dependency of the threshold laser energy for ion production on the surface tension of the liquid MALDI sample offers new insights in the mechanisms of the 
underlying desorption/ionization processes, and can be used to develop new practical guidelines for further sample optimization and theoretical models of the early processes in liquid MALDI.

In this context, spallation has been discussed as a potential MALDI desorption process for more than two decades[45] and recently by Koch et al.[36]. This discussion was initially focused on solid MALDI MS, in particular solid IR-MALDI MS due to its lower energy deposition per irradiated sample volume compared to solid UV-MALDI MS. A similarly lower laser energy deposition per irradiated sample volume (i.e. greater penetration depth) is presumably present in liquid UV-MALDI when compared again to solid UV-MALDI. Thus, spallation could be a major factor in explaining the desorption process for liquid UV-MALDI. However, the exact determination of the laser light penetration in complex liquid MALDI samples appears to be extremely difficult since the nature of a liquid droplet allows for significant surface (or nearsurface) effects due to solute-solute and solute-surface interactions which can be exacerbated by any solute accumulation at or near the surface with unknown gradients, in particular if various different solutes and solute concentrations are present. Consequently, it is equally difficult to estimate whether the thermal and acoustic (stress) confinement requirements for spallation to occur are fulfilled. Nonetheless, spallation as a model for the desorption step followed by any subsequent ionization step such as an ESI-like process as observed in liquid AP-UV-MALDI using a heated transfer tube is certainly a model that has some appeal. 


\section{Author Contributions}

All authors have given approval to the final version of the manuscript.

\section{Acknowledgements}

The authors thank Waters Corporation (Wilmslow, UK) for help with WREnS and other support in modifying the Synapt G2-Si and Valeria Casteletto (University of Reading) for help with the surface tension measurements. This work was supported by the EPSRC through grant $\mathrm{EP} / \mathrm{L} 006227 / 1$.

\section{Supplementary Material}

"Supplementary Material.docx" contains Figures S1 and S2. 


\section{References}

[1] M. Karas, F. Hillenkamp, Laser desorption ionization of proteins with molecular masses exceeding 10,000 daltons, Analytical Chemistry, 60 (1988) 2299-2301.

[2] M. Karas, D. Bachmann, F. Hillenkamp, Matrix-assisted ultraviolet laser desorption of nonvolatile compounds, Int. J. of Mass Spectrom. and Ion Proc., 78 (1987) 53-68.

[3] M. Yamashita, J.B. Fenn, Electrospray ion source. Another variation on the free-jet theme, The Journal of Physical Chemistry, 88 (1984) 4451-4459.

[4] J.B. Fenn, M. Mann, C.K. Meng, S.F. Wong, C.M. Whitehouse, Electrospray ionization for mass spectrometry of large biomolecules, Science, 246 (1989) 64-71.

[5] R. Cramer, W.J. Richter, E. Stimson, A.L. Burlingame, Analysis of Phospho- and Glycopolypeptides with Infrared Matrix-Assisted Laser Desorption and Ionization, Analytical Chemistry, 70 (1998) 4939-4944.

[6] B. Ruprecht, C. Roesli, S. Lemeer, B. Kuster, MALDI-TOF and nESI Orbitrap MS/MS identify orthogonal parts of the phosphoproteome, PROTEOMICS, 16 (2016) 1447-1456.

[7] E. Nordhoff, A. Ingendoh, R. Cramer, A. Overberg, B. Stahl, M. Karas, F. Hillenkamp, P.F. Crain, B. Chait, Matrix-assisted laser desorption/ionization mass spectrometry of nucleic acids with wavelengths in the ultraviolet and infrared, Rapid Communications in Mass Spectrometry, 6 (1992) 771-776.

[8] E. Nordhoff, M. Karas, R. Cramer, S. Hahner, F. Hillenkamp, F. Kirpekar, A. Lezius, J. Muth, C. Meier, J.W. Engels, Direct mass spectrometric sequencing of low-picomole amounts of oligodeoxynucleotides with up to 21 bases by matrix-assisted laser desorption/ionization mass spectrometry, Journal of Mass Spectrometry, 30 (1995) 99-112.

[9] E. Nordhoff, F. Kirpekar, M. Karas, R. Cramer, S. Hahner, F. Hillenkamp, K. Kristiansen, P. Roepstroff, A. Lezius, Comparison of IR- and UV-matrix-assisted laser desorption/ionization mass spectrometry of oligodeoxynucleotides, Nucleic Acids Research, 22 (1994) 2460-2465. [10] M. Erhard, H. von Dohren, P. Jungblut, Rapid typing and elucidation of new secondary metabolites of intact cyanobacteria using MALDI-TOF mass spectrometry, Nat Biotech, 15 (1997) 906-909.

[11] O.A. Koroleva, T.M. Gibson, R. Cramer, C. Stain, Glucosinolate-accumulating S-cells in Arabidopsis leaves and flower stalks undergo programmed cell death at early stages of differentiation, The Plant Journal, 64 (2010) 456-469.

[12] B. Li, D.R. Bhandari, A. Römpp, B. Spengler, High-resolution MALDI mass spectrometry imaging of gallotannins and monoterpene glucosides in the root of Paeonia lactiflora, 6 (2016) 36074.

[13] J. Sarsby, M.W. Towers, C. Stain, R. Cramer, O.A. Koroleva, Mass spectrometry imaging of glucosinolates in Arabidopsis flowers and siliques, Phytochemistry, 77 (2012) 110-118.

[14] L. Charles, MALDI of synthetic polymers with labile end-groups, Mass Spectrometry Reviews, 33 (2014) 523-543.

[15] H.J. Räder, T.-T.-T. Nguyen, K. Müllen, MALDI-TOF Mass Spectrometry of Polyphenylene Dendrimers up to the Megadalton Range. Elucidating Structural Integrity of Macromolecules at Unrivaled High Molecular Weights, Macromolecules, 47 (2014) 1240-1248. [16] A.N. Krutchinsky, A.V. Loboda, V.L. Spicer, R. Dworschak, W. Ens, K.G. Standing, Orthogonal injection of matrix-assisted laser desorption/ionization ions into a time-of-flight spectrometer through a collisional damping interface, Rapid Communications in Mass Spectrometry, 12 (1998) 508-518. 
[17] A.V. Loboda, A.N. Krutchinsky, M. Bromirski, W. Ens, K.G. Standing, A tandem quadrupole/time-of-flight mass spectrometer with a matrix-assisted laser desorption/ionization source: design and performance, Rapid communications in mass spectrometry : RCM, 14 (2000) 1047-1057.

[18] V.V. Laiko, M.A. Baldwin, A.L. Burlingame, Atmospheric pressure matrix-assisted laser desorption/ionization mass spectrometry, Analytical Chemistry, 72 (2000) 652-657.

[19] P.B. O'Connor, C.E. Costello, A high pressure matrix-assisted laser desorption/ionization Fourier transform mass spectrometry ion source for thermal stabilization of labile biomolecules, Rapid Communications in Mass Spectrometry, 15 (2001) 1862-1868.

[20] P.B. O’Connor, E. Mirgorodskaya, C.E. Costello, High pressure matrix-assisted laser desorption/ionization fourier transform mass spectrometry for minimization of ganglioside fragmentation1, J. Am. Soc. Spectrom., 13 (2002) 402-407.

[21] A.L. Yergey, J.R. Coorssen, P.S. Backlund, P.S. Blank, G.A. Humphrey, J. Zimmerberg, J.M. Campbell, M.L. Vestal, De novo sequencing of peptides using MALDI/TOF-TOF, J. Am. Soc. Spectrom., 13 (2002) 784-791.

[22] A.J. Hoteling, K.G. Owens, Improved PSD and CID on a MALDI TOFMS, J. Am. Soc. Mass Spectrom., 15 (2004) 523-535.

[23] K. Wiangnon, R. Cramer, Liquid MALDI MS Analysis of Complex Peptide and Proteome Samples, Journal of Proteome Research, 15 (2016) 2998-3008.

[24] R. Cramer, S. Corless, The nature of collision-induced dissociation processes of doubly protonated peptides: comparative study for the future use of matrix-assisted laser desorption/ionization on a hybrid quadrupole time-of-flight mass spectrometer in proteomics, Rapid Communications in Mass Spectrometry, 15 (2001) 2058-2066.

[25] S. König, O. Kollas, K. Dreisewerd, Generation of Highly Charged Peptide and Protein Ions by Atmospheric Pressure Matrix-Assisted Infrared Laser Desorption/Ionization Ion Trap Mass Spectrometry, Analytical Chemistry, 79 (2007) 5484-5488.

[26] S. Trimpin, E.D. Inutan, T.N. Herath, C.N. McEwen, Laserspray Ionization, a New Atmospheric Pressure MALDI Method for Producing Highly Charged Gas-phase Ions of Peptides and Proteins Directly from Solid Solutions, Mol Cell Proteomics, 9 (2010) 362-367. [27] R. Cramer, A. Pirkl, F. Hillenkamp, K. Dreisewerd, Liquid AP-UV-MALDI enables stable ion yields of multiply charged peptide and protein ions for sensitive analysis by mass spectrometry, Angew. Chem. Int. Ed. Engl., 52 (2013) 2364-2367.

[28] R. Cramer, S. Corless, Liquid ultraviolet matrix-assisted laser desorption/ionization - mass spectrometry for automated proteomic analysis, PROTEOMICS, 5 (2005) 360-370.

[29] M. Palmblad, R. Cramer, Liquid matrix deposition on conductive hydrophobic surfaces for tuning and quantitation in UV-MALDI mass spectrometry, J. Am. Soc. Mass Spectrom., 18 (2007) 693-697.

[30] M.W. Towers, J.E. McKendrick, R. Cramer, Introduction of 4-Chloro- $\alpha$-cyanocinnamic Acid Liquid Matrices for High Sensitivity UV-MALDI MS, Journal of Proteome Research, 9 (2010) 1931-1940.

[31] N.D. Chubatyi, C.N. McEwen, Improving the Sensitivity of Matrix-Assisted Ionization (MAI) Mass Spectrometry Using Ammonium Salts, J. Am. Soc. Spectrom., 26 (2015) 16491656.

[32] P. Ryumin, J. Brown, M. Morris, R. Cramer, Investigation and optimization of parameters affecting the multiply charged ion yield in AP-MALDI MS, Methods, 104 (2016) 11-20. 
[33] P. Ryumin, J. Brown, M. Morris, R. Cramer, Protein identification using a nanoUHPLCAP-MALDI MS/MS workflow with CID of multiply charged proteolytic peptides, International Journal of Mass Spectrometry, 416 (2017) 20-28.

[34] D.W. Armstrong, L.-K. Zhang, L. He, M.L. Gross, Ionic Liquids as Matrixes for MatrixAssisted Laser Desorption/Ionization Mass Spectrometry, Analytical Chemistry, 73 (2001) 36793686.

[35] A. Tholey, E. Heinzle, Ionic (liquid) matrices for matrix-assisted laser desorption/ionization mass spectrometry - applications and perspectives, Analytical and Bioanalytical Chemistry, 386 (2006) 24-37.

[36] A. Koch, A. Schnapp, J. Soltwisch, K. Dreisewerd, Generation of multiply charged peptides and proteins from glycerol-based matrices using lasers with ultraviolet, visible and near-infrared wavelengths and an atmospheric pressure ion source, International Journal of Mass Spectrometry, 416 (2017) 61-70.

[37] P. Ryumin, J. Brown, M. Morris, R. Cramer, Optimized multiply charged ion production by liquid MALDI - From large intact protein ions to bottom-up proteomics using LC-MALDI MS/MS, 64th ASMS Conference on Mass SpectrometrySan Antonio, TX, USA, 2016. [38] W.M. Haynes, D.R. Lide, T.J. Bruno, Handbook of Chemistry and Physics, Taylor \& Francis Group, LLC, Boca Raton, 2016.

[39] M.C. Chambers, B. Maclean, R. Burke, D. Amodei, D.L. Ruderman, S. Neumann, L. Gatto, B. Fischer, B. Pratt, J. Egertson, K. Hoff, D. Kessner, N. Tasman, N. Shulman, B. Frewen, T.A. Baker, M.-Y. Brusniak, C. Paulse, D. Creasy, L. Flashner, K. Kani, C. Moulding, S.L. Seymour, L.M. Nuwaysir, B. Lefebvre, F. Kuhlmann, J. Roark, P. Rainer, S. Detlev, T. Hemenway, A. Huhmer, J. Langridge, B. Connolly, T. Chadick, K. Holly, J. Eckels, E.W. Deutsch, R.L. Moritz, J.E. Katz, D.B. Agus, M. MacCoss, D.L. Tabb, P. Mallick, A cross-platform toolkit for mass spectrometry and proteomics, Nat Biotech, 30 (2012) 918-920.

[40] A.A. Goloborodko, L.I. Levitsky, M.V. Ivanov, M.V. Gorshkov, Pyteomics—a Python Framework for Exploratory Data Analysis and Rapid Software Prototyping in Proteomics, J. Am. Soc. Mass Spectrom., 24 (2013) 301-304.

[41] T. Bald, J. Barth, A. Niehues, M. Specht, M. Hippler, C. Fufezan, pymzML - Python module for high throughput bioinformatics on mass spectrometry data. , Bioinformatics, 28 (2012) 1052-1053.

[42] J.D. Hunter, Matplotlib: A 2D Graphics Environment, Computing in Science \& Engineering, 9 (2007) 90-95.

[43] O.J. Hale, R. Cramer, Collision-induced dissociation of doubly-charged barium-cationized lipids generated from liquid samples by atmospheric pressure matrix-assisted laser desorption/ionization provides structurally diagnostic product ions, Analytical and Bioanalytical Chemistry, in press (2016).

[44] R. Juraschek, T. Dulcks, M. Karas, Nanoelectrospray - More than just a minimized-flow electrospray ionization source, J. Am. Soc. Spectrom., 10 (1999) 300-308.

[45] R. Cramer, R.F. Haglund, F. Hillenkamp, Matrix-assisted laser desorption and ionization in the $\mathrm{O}-\mathrm{H}$ and $\mathrm{C}=\mathrm{O}$ absorption bands of aliphatic and aromatic matrices: dependence on laser wavelength and temporal beam profile, International Journal of Mass Spectrometry, 169 (1997) 51-67. 


\section{Highlights}

- New liquid AP-MALDI matrices for protein and peptide analysis were developed.

- Electrospray-like MS spectra of proteins as high as $\sim 80 \mathrm{kDa}$ were detected by liquid APUV-MALDI MS.

- Chromophores and support liquids as well as plume desolvation conditions were found to play a crucial role in the detection of high mass proteins by liquid MALDI MS.

- A linear dependency between laser threshold energy for analyte ionization and surface tension of the liquid MALDI sample was established.

\section{Graphical abstract}

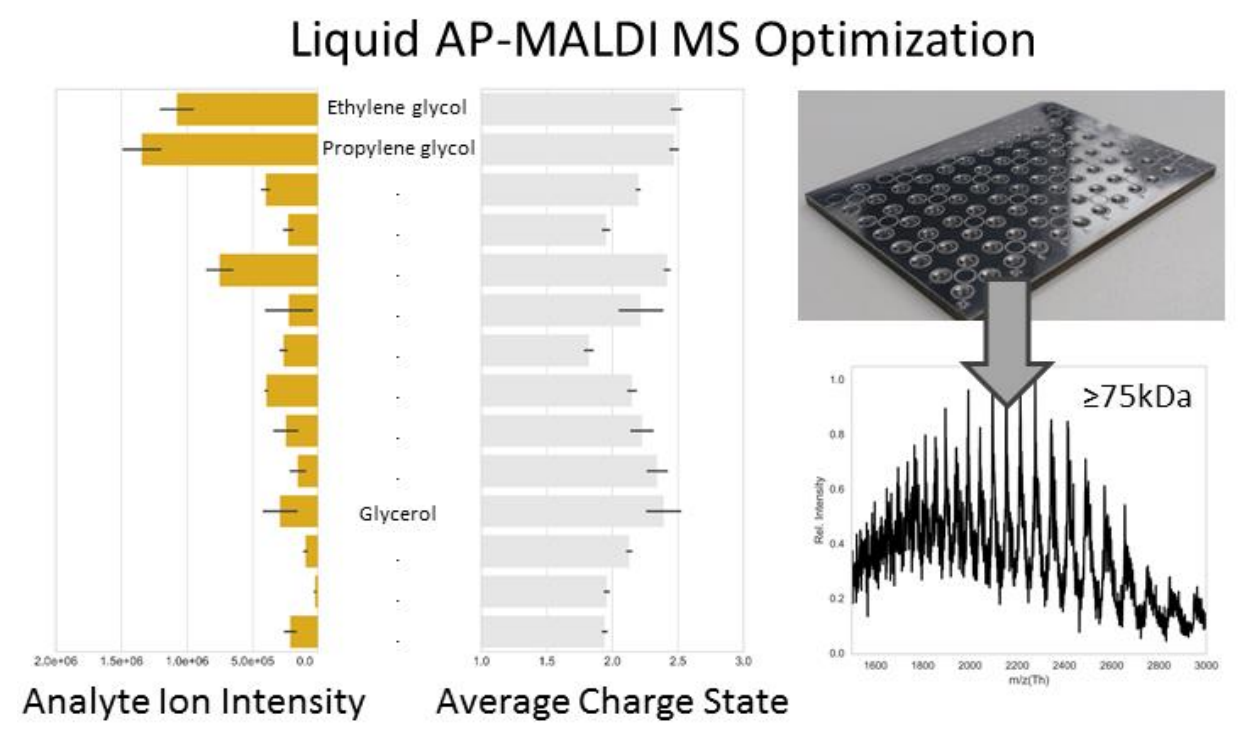




\title{
The composition of liquid atmospheric pressure
}

\author{
matrix-assisted laser desorption/ionization
}

\section{matrices and its effect on ionization in mass}

\section{spectrometry}

\author{
Pavel Ryumin and Rainer Cramer*
}

Department of Chemistry, University of Reading, Reading, Berkshire, RG6 6AD, UK

\section{Table of Contents:}

Page S-2 Figure S1: Liquid AP-MALDI MS of human carbonic anhydrase I (10 pmol protein loading) in response to different viscous support liquids.

Page S-3 Figure S2: Liquid AP-MALDI MS of bovine serum albumin (12.5 pmol protein loading) in response to different viscous support liquids. 

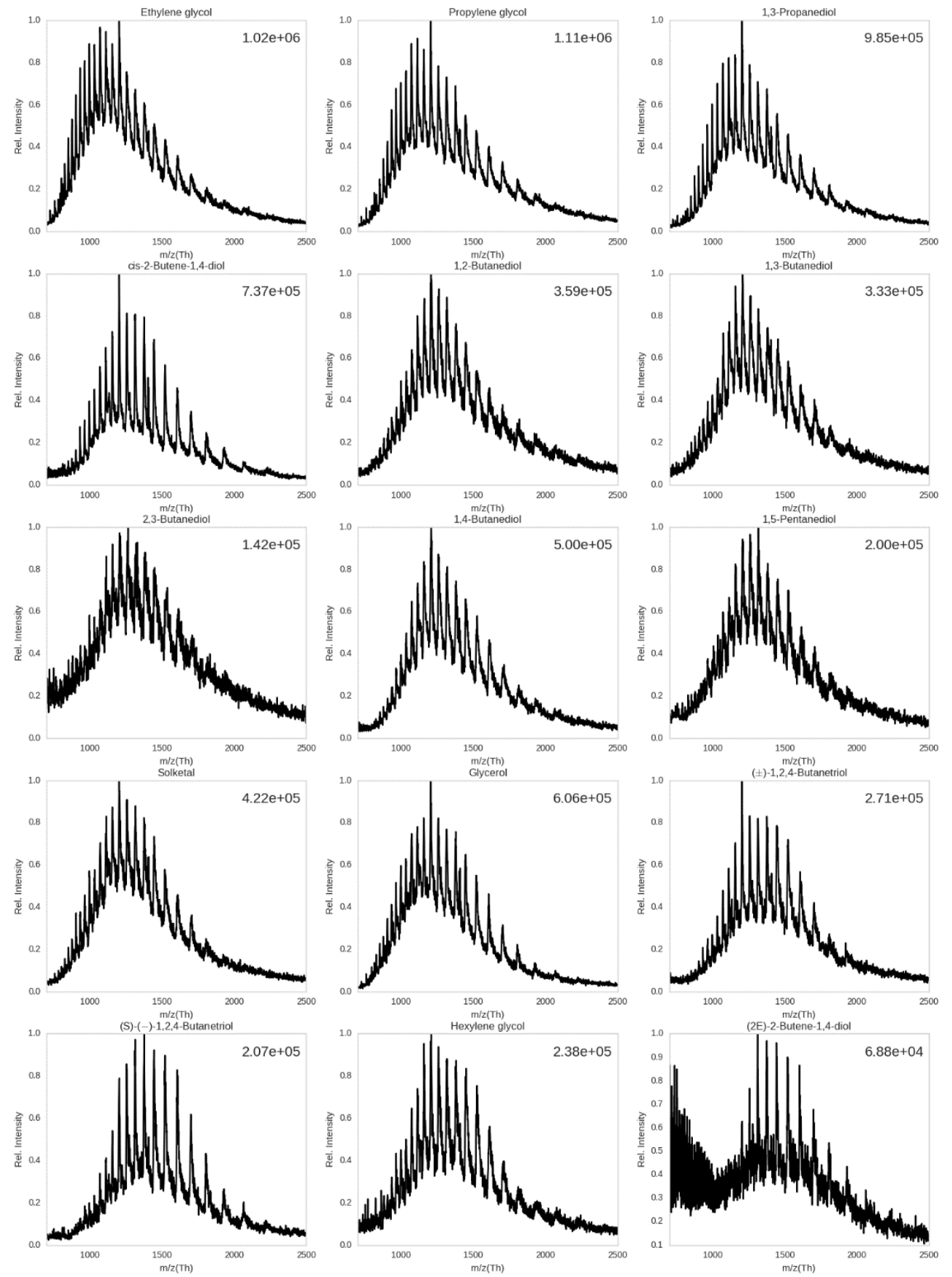

Figure S1: Liquid AP-MALDI MS of human carbonic anhydrase I (10 pmol protein loading) in response to different viscous support liquids. 

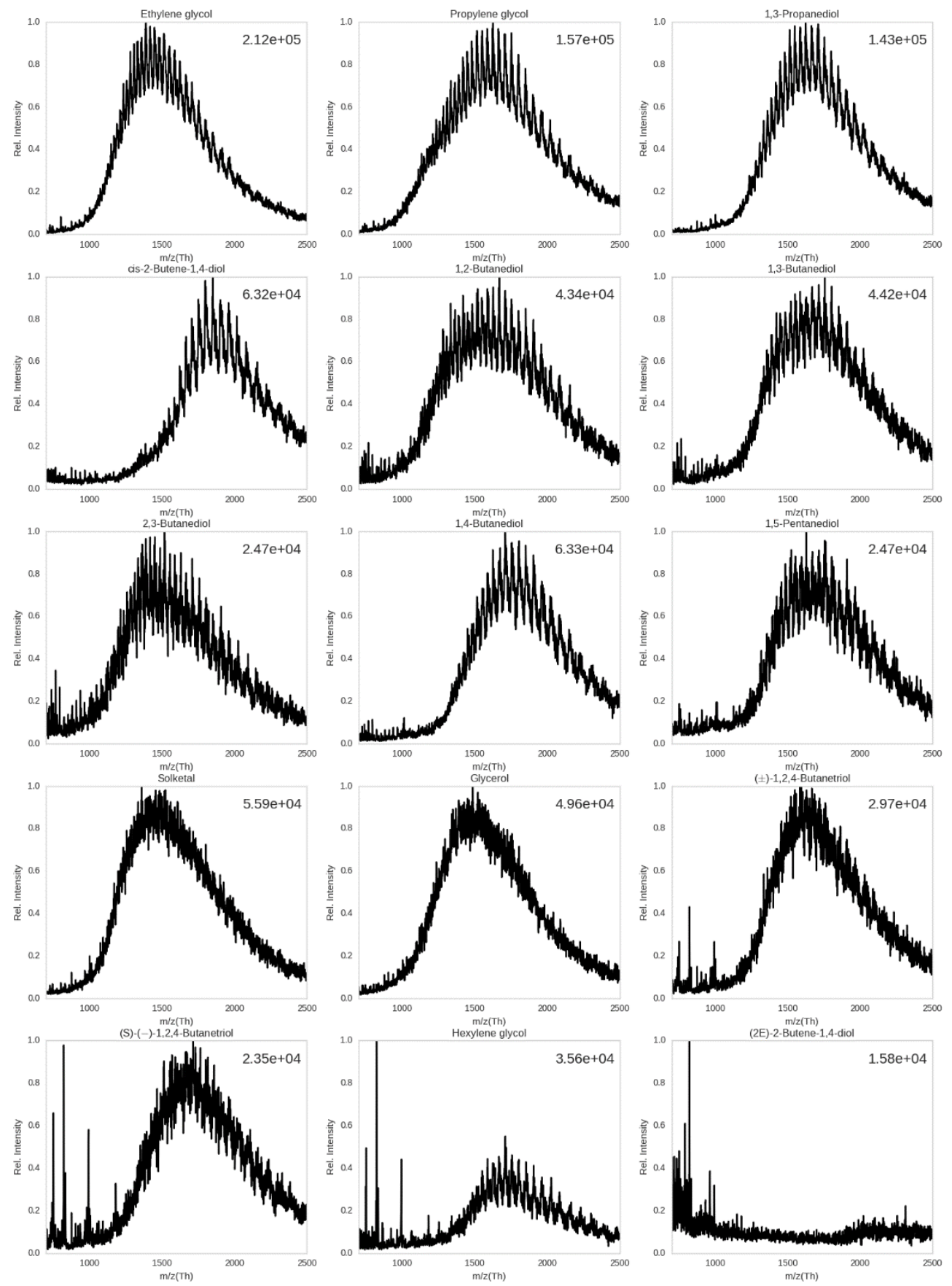

Figure S2: Liquid AP-MALDI MS of bovine serum albumin (12.5 pmol protein loading) in response to different viscous support liquids. 